\title{
LA ACREDITACIÓN UNIVERSITARIA EN EL PERÚ
}

\section{José Ignacio López Soria $(*)$}

SÍNTESIS: Este trabajo da cuenta de los aspectos más significativos de la situación actual de la acreditación de la formación universitaria en el Perú, comenzando por las facultades y escuelas de Medicina, dando cuenta después de los avances producidos en acreditación de los estudios universitarios de pregrado y presentando algunas iniciativas relacionadas con la acreditación de los estudios de postgrado, para terminar con una reseña de la legislación vigente sobre evaluación y acreditación. Hay que tener en cuenta que se trata en general de un proceso en marcha, $y$, por tanto, la información recogida aquí puede quedar superada por los hechos en poco tiempo.

SÍNTESE: Este trabalho contempla os aspectos mais significativos da situação atual da acreditação da formação universitária no Peru, começando pelas faculdades e escolas de Medicina, os avanços produzidos na acreditação dos estudos universitários de pré-graduação e apresenta algumas iniciativas relacionadas com a acreditação dos estudos de pósgraduação, para terminar com uma resenha da legislação vigente sobre avaliação e acreditação. Considerando-se que se trata, em geral, de um processo em andamento, a informação coletada aqui pode, portanto, ficar, em pouco tempo, superada pelos fatos.

(*) Director de la Oficina Regional en Lima de la Organización de Estados Iberoamericanos (OEI). 


\section{INTRODUCCIÓN}

Es sabido que en el Perú la cultura y la práctica modernas de la acreditación son muy recientes, incluso más que en los países vecinos. Decimos «modernas» porque, en realidad, la acreditación nos viene de la época colonial y estuvo vigente en buena parte del siglo XIX.

Cuando todavía las experiencias y los saberes necesarios para el ejercicio profesional no se habían constituido con claridad ni existían caminos escolarizados para la formación de profesionales, existía sin embargo una institución, el Cosmografiato, que tenía entre otras responsabilidades la de acreditar o dar fe pública de las competencia de una persona para el ejercicio de un determinado oficio u ocupación. En Lima la acreditación era dispensada por el cosmógrafo mayor, y en provincias por sus representes. Tal atribución fue luego transferida a los municipios para el caso de los oficios, convirtiéndose en una mera licencia para su desempeño, y al Cuerpo de Ingenieros y Arquitectos del Estado para los egresados de estas carreras y para sus ayudantes. Esta última institución supuso después el corpus de competencias de las que debían estar provistos los que deseasen ser reconocidos como ingenieros o ayudantes, e, igualmente, otro para arquitectos y para sus ayudantes. Incluso se puso en práctica un camino para adquirir esas competencias a través del trabajo, yun sistema de exámenes para verificar si los candidatos poseían las mencionadas competencias.

Todo este andamiaje para la provisión y acreditación de competencias profesionales fue quedando en desuso en la segunda mitad del siglo XIX, cuando se escolarizó la formación de técnicos con la creación de las escuelas de artes y oficios; la de científicos, juristas, médicos y literatos, con la constitución de las respectivas facultades en la Universidad de San Marcos y en otras universidades; la de ingenieros, arquitectos y peritos agrimensores con la creación de la Escuela de Ingenieros en 1876; y la de profesionales del trabajo agrícola con el nacimiento de la Escuela de Agricultura en 1901. Desde entonces, la acreditación se confunde con la concesión del título profesional por parte de las instituciones formativas que cuenten con la correspondiente autorización. En algunos casos: médicos, ingenieros, arquitectos, abogados, contadores públicos, etc., al título universitario es necesario añadir el registro en el respectivo colegio profesional para el ejercicio de la profesión. 
En las páginas que siguen daremos cuenta de los aspectos más destacados de la acreditación de la formación universitaria en el Perú en la actualidad, comenzando por las facultades y escuelas de Medicina, dando cuenta después de los avances producidos en acreditación de los estudios universitarios de pregrado y presentando algunas iniciativas relacionadas con la acreditación de los estudios de postgrado, para finalizar con una reseña de la legislación vigente sobre evaluación y acreditación.

\section{ACREDITACIÓN DE FACULTADES Y ESCUELAS DE MEDICINA}

La historia oficial de la acreditación de estudios de Medicina

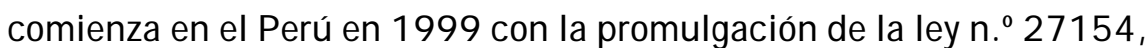
que institucionaliza la acreditación de las facultades y escuelas de Medicina. Sin embargo, tal promulgación fue fruto de un proceso que se inició algunos años antes.

Recogiendo una tradición que se remonta a 1910 (informe de Abraham Flexner sobre la proliferación irracional de facultades de Medicina en Estados Unidos) y que cuaja en 1943 (creación del Comité de Enlace para la Educación Médica en Estados Unidos), la Asociación Peruana de Facultades de Medicina (ASPEFAM), fundada en 1964, organizó en 1992 el I Seminario de Acreditación de Facultades de Medicina, contando con la participación de todos los centros de formación en la materia y con la asesoría de la Organización Panamericana de la Salud (OPS).

Tras este esfuerzo inicial - anota Piscoya (2002)-, el tema de la acreditación siguió siendo debatido en boletines y en congresos especializados. En 1998 el Ministerio de Salud convocó a las Universidades de San Marcos y Cayetano Heredia, a la Academia Nacional de Medicina, a la ASPEFAM y al Colegio Médico, para que presentaran una propuesta de ley de acreditación de las facultades de Medicina.

La citada ley $n .027154$ garantiza la calidad y la idoneidad de la formación de los médicos y la atención sanitaria a la comunidad; crea la Comisión para la Acreditación de Facultades o Escuelas de Medicina Humana (CAFME); determina su composición, y le asigna como funciones la elaboración de los estándares mínimos para la acreditación, así como la administración del proceso y su supervisión y evaluación permanentes; 
suspende la creación de nuevas facultades o escuelas de Medicina; determina el proceso al que deben someterse los centros formativos que no alcancen los estándares mínimos exigidos para la acreditación; adscribe la CAFME al Ministerio de Salud concediéndole autonomía técnica y administrativa, y le da un plazo de 120 días para que elabore una propuesta de normas para establecer los estándares mínimos de acreditación de los centros educativos de Medicina.

En diciembre de 2000 se aprobó por Decreto Supremo 005 2000-SA el Reglamento de la ley $n .027154$. En él se establecen los requisitos y las condiciones generales a los que debe sujetarse el funcionamiento de las facultades y escuelas de Medicina para garantizar la idoneidad y la calidad en la formación de médicos cirujanos, así como las normas para la conformación y el funcionamiento de la CAFME.

En cuanto a ésta última, el Reglamento establece que se constituye sobre la base de un representante de cada una de las siguientes instituciones: Ministerio de Salud, Ministerio de Educación, Asamblea Nacional de Rectores, Consejo Nacional para la Autorización del Funcionamiento de Universidades, y Colegio Médico del Perú. Sus funciones son: elaborar los estándares mínimos para la acreditación; formular las normas y los procedimientos de acreditación; acreditar a facultades y a escuelas de Medicina; establecer plazos y procedimientos para la adecuación de las no acreditadas; supervisar el proceso de adecuación; evaluar y emitir opinión previa sobre la creación de nuevos centros de formación; además, elaborar el presupuesto anual y sus normas internas de funcionamiento.

Finalmente, el Reglamento concede a las facultades y a las escuelas ya autorizadas un plazo máximo de un año, contado a partir de la aprobación oficial de los estándares mínimos de acreditación, para que dichos centros de formación soliciten su acreditación. Si no lo hicieren, la CAFME queda autorizada para verificar de oficio si cumplen o no con los estándares establecidos.

En enero de 2001 la Resolución Suprema n. o 013-2001-SA aprobó los estándares mínimos para la acreditación de facultades y de escuelas de Medicina.

Los estándares mínimos de acreditación tendrán que evaluar los siguientes aspectos: organización académica, currículo, proceso de admisión, cuerpo docente, prevención de riesgos de salud asociados a las 
prácticas preprofesionales, competencias adquiridas por los estudiantes, metodología de enseñanza-aprendizaje, organización administrativa, servicios académicos complementarios, así como infraestructura física y equipamiento.

Aprobados los estándares mínimos, las facultades y las escuelas de Medicina que contaran con una o más promociones de egresados tuvieron un año, hasta enero de 2002, para solicitar la acreditación ante la CAFME, debiendo iniciar antes el proceso de autoevaluación conforme a los estándares mínimos aprobados. Conseguida la acreditación, ésta debe ser renovada cada cinco años.

Las universidades con autorización de funcionamiento definitivo, pero cuyas facultades o escuelas de Medicina no cuenten aún con egresados, deben someterse a visitas de verificación por parte de la CAFME para comprobar el cumplimiento de los estándares mínimos, y cada año deben informarla sobre el cumplimiento de dichos estándares.

Para las facultades y escuelas de Medicina en universidades con autorización de funcionamiento provisional, corresponde al CONAFU (EI Consejo Nacional para la Autorización del Funcionamiento de Universidades), en coordinación con la CAFME, verificar el cumplimiento de los estándares mínimos mientras dure la situación de provisionalidad. CONAFU, además, debe tener en cuenta los estándares mínimos cuando evalúe los proyectos de creación o de fusión de universidades que incluyan facultades o escuelas de Medicina.

Por último, para el caso de las universidades con autorización definitiva de funcionamiento, corresponde a la CAFME autorizar la creación de nuevas facultades o escuelas de Medicina.

En enero de 2002, cuando el proceso de acreditación estaba en pleno desarrollo, se aprobó el Manual de Procedimientos de la Comisión de Acreditación. Este Manual establece los procedimientos específicos para cada uno de los casos mencionados.

Aprobados los estándares mínimos en enero de 2001, comenzó de inmediato el proceso de acreditación. En 1958 había en el Perú tres facultades de Medicina, todas ellas públicas. De 1960 a 1990 se crearon otras 11 (cuatro privadas y siete públicas), y de 1990 a 1998 se establecieron 14 nuevas facultades (ocho privadas y seis públicas). Con el fin de detener este irracional crecimiento, en 1999 se suspendió 
la creación de nuevas facultades. La acreditación se aplica, pues, a los 28 centros académicos ( 12 privados y 16 públicos) que existen actualmente en el país para la formación de médicos.

En 2001 comenzaron las visitas a las facultades para verificar el cumplimiento de los estándares. De las 19 facultades con una o más promociones de egresados, siete (tres privadas y cuatro públicas) consiguieron su acreditación en 2002 por cinco años, mientras que 12 (tres privadas y nueve públicas) lo hicieron en 2003 . Las nueve restantes no tenían aún egresados cuando comenzó el proceso de acreditación, y, por tanto, quedaron sujetas a la verificación del cumplimiento de los estándares según el grado de desarrollo de cada una. La verificación arrojó el siguiente resultado: siete (cinco privadas y dos públicas) cumplieron con los estándares correspondientes, y dos (una privada y otra pública) no lo hicieron; por eso quedaron sujetas a nuevas verificaciones, e, incluso, expuestas a que la CAFME pudiera solicitar a la Asamblea Nacional de Rectores la suspensión de nuevas inscripciones.

Logros indiscutibles de laCAFME han sido: 1. Haber incorporado a las 28 facultades y escuelas de Medicina, a los principales colectivos de médicos y a instituciones de salud, al proceso de elaboración de los instrumentos de evaluación y acreditación, que, además, están prolijamente desarrollados; 2 . Estar impulsando la cultura de la calidad en los medios de la formación médica; 3. Haber puesto en la agenda política y universitaria la necesidad de la acreditación; y, 4. Haber desarrollado criterios y procedimientos que pueden servir de antecedentes para la extensión de la práctica de la acreditación a otras áreas profesionales.

Sin embargo, se le critica que confunda acreditación con certificación, reduciendo la condición para la acreditación al cumplimiento de los requisitos exigidos para la autorización o continuación del funcionamiento; que haya fijado estándares de calidad no equivalentes a los exigidos internacionalmente; y que se haya convertido en instancia de legitimación de la mediocridad y de la falta de idoneidad, al acreditar prácticamente a todas las facultades existentes.

De ser ciertas, estas críticas echarían por tierra el sentido mismo de la acreditación, para convertirla en un expediente burocrático que en poco o en nada contribuiría a garantizar la calidad de la oferta educativa en las áreas de la salud. En su descargo, hay que decir que se 
trata de la primera experiencia de acreditación en el Perú, que debe alimentar los actuales procesos de acreditación.

\section{ACREDITACIÓN DE ESTUDIOS UNIVERSITARIOS DE PREGRADO}

Respondiendo a la cultura de la acreditación que comenzaba a extenderse en los ambientes universitarios, la Asamblea Nacional de Rectores ( ANR) constituyó el 1 de febrero de 2002 la Comisión Nacional de Rectores para la Acreditación Universitaria (CNRAU). Además de revisar y de hacer propuestas de estándares de acreditación y de evaluación de las universidades, la Comisión se propone difundir la necesidad de la evaluación, y facilitar y promover que se instalen mecanismos permanentes de autoevaluación. Para ello, la CNRAU apoya las actividades de las universidades dirigidas a mejorar la calidad de la educación que imparten, y propicia y promueve talleres de motivación para incorporar la cultura de la autoevaluación. La Comisión cuenta con el apoyo de la Dirección General de Investigación y Acreditación Universitaria, que, por otra parte, se encarga de mantener la información relacionada con el proceso de mejoramiento de la calidad en las universidades.

La CNRAU está compuesta por un Comité Central integrado por cinco rectores, y por un Comité Ampliado de ocho rectores, y se apoya en una Comisión Técnica de la ANR, constituida por la Dirección General de Desarrollo Académico y Capacitación, Ia Dirección General de Investigación y Acreditación Universitaria, la Oficina de Asesoría Académica y la Secretaría General.

En el 2002 la CNRAU llevó a cabo una serie de reuniones en las que se acordó, entre otras cosas: contratar a un experto para desarrollar la filosofía y los conceptos básicos de la acreditación; incorporar a especialistas por áreas profesionales para la elaboración de un plan de trabajo; informar a los colegios profesionales sobre la creación y el funcionamiento de la CNRAU y solicitarles sus sugerencias; difundir los avances en la página web de la ANR; proponer a las universidades el establecimiento de una Oficina de Evaluación y Acreditación, con representantes de las diversas facultades; remitir el documento «Propuesta de Acreditación-Programas de Postgrado» a las escuelas de postgrado de las universidades, a fin de que éstas envíen sus iniciativas para perfeccionar el documento; organizar un foro de escuelas de postgrado para discutir la propuesta de acreditación; organizar un ciclo 
de conferencias y de seminarios sobre autoevaluación en las áreas de ingeniería, humanidades y ciencias sociales, ciencias puras y ciencias de la salud, con la participación de la CAFME y de Fernando Ocampo, experto mexicano en acreditación en áreas de ingeniería; impulsar un seminario sobre evaluación y acreditación en educación, con el propósito de analizar el proyecto de ley destinado a institucionalizar la acreditación de las facultades de educación; solicitar que la autorización para el funcionamiento de nuevas escuelas de postgrado se otorgue tras la aprobación de los estándares mínimos de acreditación; hacer seguimiento de los procesos de autoevaluación y acreditación de las universidades; constituir grupos de trabajo para elaborar estándares por áreas afines; y poner a punto programas regionales de capacitación para evaluadores.

La CNRAU continuó sus trabajos en el 2003 en favor de la promoción y el apoyo a las universidades, para la difusión de la cultura de la calidad y para la ampliación y profundización de los conceptos sobre autoevaluación y mejora continua con fines de acreditación universitaria. Para ello, organizó o auspició la realización de talleres y de seminarios en las universidades, que han contribuido a difundir la cultura de la calidad, a profundizar y a socializar los conceptos básicos de la acreditación, y a elaborar herramientas prácticas para iniciar los procesos de autoevaluación, preparando así las condiciones para la acreditación.

Fruto de estos esfuerzos fue la creación, en el propio 2003, de oficinas de evaluación y acreditación en 28 universidades públicas y 29 privadas.

Además, la CNRAU considera como un tema de especial relevancia en su plan de trabajo la elaboración de estándares de calidad. Con el apoyo de la Dirección General de Desarrollo Académico de la ANR y con la participación activa de los decanos y de los jefes de las escuelas profesionales, se han elaborado y aprobado los estándares de calidad de las especialidades de Educación, Derecho y Estomatología con sus respectivos postgrados, así como los estándares mínimos deautoevaluación con fines de acreditación de las ingenierías. Estos estándares ya están siendo aplicados por algunas universidades, y sus avances constituyen una valiosa experiencia para la formulación de los de las especialidades que faltan.

De todos los procesos puestos en marcha, la ANR está reuniendo una importante masa de informes, de documentos, de investigaciones y de publicaciones sobre autoevaluación y acreditación, que pone a 
disposición de las universidades y de los grupos interesados en el tema. Por otra parte, las oficinas especializadas de la ANR brindan información y asesoría a todas las personas y a todas las instituciones preocupadas por el mejoramiento de la calidad educativa y por las prácticas de la acreditación.

Un paso importante en el proceso de introducción de la acreditación ha sido la creación por la ANR del Consejo Nacional de AcreditaciónUnivensitaria.E128 deoctubre de 2003 laANR, después de comprobar el interés que existe de preparar a las universidades para que a mediano plazo puedan solicitar la acreditación correspondiente y de dar a conocer los avances producidos, aprobó la propuesta de creación del Consejo Nacional de Acreditación Universitaria, y acordó elevar al Congreso de la República, para su ratificación, un proyecto de ley en este sentido. Mientras tanto, la ANR, por resolución de enero de 2004, creó el Consejo como organismo autónomo, técnico, administrativo y con recursos propios, encargado de elaborar las normas para llevar a cabo los procesos de acreditación de la educación universitaria, así como la administración, la supervisión y la evaluación permanentes de las universidades.

El Consejo Nacional de Acreditación Universitaria formará parte de la estructura de laANR, y estará integrado por dos representantes de los Colegios Profesionales, por dos de la CONFIEP (Confederación Nacional de Empresarios Privados), por un representante del Ministerio de Educación, por otro de la Asamblea Nacional de Rectores, por dos representantes del Ministerio de la Producción, por uno del Ministerio de Economía, por uno de los Consejos Regionales Universitarios, y por un representante del Consejo Nacional de Ciencia y Tecnología.

En cuanto al Programa de Formación de Evaluadores, los avances de la ANR han sido menores. Para su elaboración se ha contado con el apoyo de organismos de acreditación de países cercanos: Ia CN Ade Colombia y el CONEAU de Argentina.

Por otra parte, Ia ANR, aprovechando la apertura de la Comunidad Andina (CA) a una integración que supera lo estrictamente comercial, está tratando de interesar a su Secretaría General para que actúe en procesos de acreditación con perspectiva regional. Además de solicitar el apoyo de lacA para el desplazamiento de expertos, sobre todo en el área de formación de evaluadores, la ANR ha propuesto la elaboración de un documento base para la homologación de títulos y para la acreditación 
de carreras, y está empeñada en crear el Comité Andino de Acreditación de Profesionales. Estas propuestas se dan en un contexto en el que son cada vez más intensos los intercambios y la movilidad de capitales, de productos, de instituciones, de personas, de procedimientos, de imágenes y de símbolos entre los cinco países que conforman la Comunidad Andina: Bolivia, Colombia, Ecuador, Perú y Venezuela.

En el 2004, la ANR ha seguido trabajando en la aplicación y en el perfeccionamiento de los estándares de autoevaluación ya aprobados, y en la elaboración de otros para algunas de las carreras que faltan: Administración, Antropología, Arquitectura, Arte, Contabilidad, Economía, Psicología y Sociología, entre otras. Paralelamente, está empeñada en la elaboración de guías para la realización de los procesos de autoevaluación y para la formulación de proyectos de mejoramiento sobre la base de tal autoevaluación, y en el desarrollo de actividades relacionadas con la formación de evaluadores.

Por otra parte, Ia ANR participa en la Red Iberoamericana de Evaluación y Acreditación de la Calidad de la Educación Superior (RIACES), que tiene como misiones extender la cultura de la calidad y promover la elaboración y la aplicación de herramientas para la evaluación y la acreditación de la educación superior en el mundo iberoamericano.

Finalmente, como aporte importante de la ANR, hay que mencionar la contribución de la experta en acreditación y responsable de la Dirección General de Desarrollo Académico y Capacitación, Doris Maraví, que ha sido encargada de la elaboración del glosario de evaluación y acreditación universitaria que tiene en prensa la ANECA de España. Para la elaboración de dicho glosario, la OEI ha conseguido también la participación de expertos peruanos y de otros ámbitos de Iberoamérica.

En consonancia, y con frecuencia en coordinación con los avances de laANR en materia de acreditación, las universidades peruanas han comenzado a movilizarse para difundir la cultura de la calidad, y para preparar las condiciones necesarias destinadas a iniciar los procesos de autoevaluación, de evaluación externa y de acreditación.

Los decanos de las facultades de Educación realizan reuniones anuales, desde 1993, para discutir los estándares mínimos de calidad. La aprobación de esos estándares no se ha producido todavía. 
En 1996 se reunieron cuatro universidades privadas de Lima (la Pontificia Universidad Católica del Perú, la Universidad de Lima, la Universidad Peruana Cayetano Heredia y la Universidad del Pacífico) para formar el «Consorcio de Universidades». Dicho consorcio ha tenido como meta, desde sus inicios, el mejoramiento de la enseñanza, yalberga la intención de llegar a la acreditación de sus carreras.

Desde 1996 varias universidades (Pontificia Universidad Católica del Perú, Universidad Nacional de Ingeniería, U niversidad Nacional Federico Villarreal, Universidad de Piura, y Universidad Peruana de Ciencias Aplicadas) están trabajando para lograr la acreditación de sus carreras de Ingeniería Civil en la Accreditation Board for Engineering and Technology ( $\mathrm{ABET}$ ), una institución privada norteamericana. Recientemente se estudia también la acreditación de las carreras de Electricidad, de Electrónica y de Sistemas en la propia ABET de las Universidades Nacional de Ingeniería, Pontificia U niversidad Católica del Perú, Universidad Peruana de Ciencias Aplicadas y Universidad San Martín de Porres.

Las principales universidades del país han constituido durante los últimos dos o tres años oficinas centrales de control de calidad o de acreditación. Por lo general, el trabajo de dichas oficinas está centrado en difundir dentro de su universidad información sobre las necesidades de la calidad educativa y de la acreditación, tratando de sensibilizar a sus docentes, organizando seminarios y publicando documentos (por ejemplo, la oficina respectiva de la Universidad Nacional de Ingeniería ha publicado seis volúmenes que reúnen libros y artículos sobre calidad y acreditación). Existen también importantes publicaciones sobre el tema editadas por la Universidad Peruana Cayetano Heredia.

\section{ACREDITACIÓN DE LOS ESTUDIOS DE POSTGRADO}

Sumándose a los esfuerzos que vienen haciendo diversas redes institucionales, el Consejo Nacional de Ciencia y Tecnología (CONCYTEC) se propuso también contribuir a la difusión de la cultura de la calidad educativa, sobre todo con respecto a los estudios de postgrado.

Es sabido que los estudios de postgrado, cuya oferta se ha incrementado mucho en los últimos años, adolecen de una serie de deficiencias. Según un informe del CONCYTEC, las principales son las 
siguientes: desadecuación entre la oferta formativa y las necesidades del desarrollo, falta de programas de investigación que sirvan de sustento a los programas de postgrado, escasa producción científica, bajas tasas de graduación en relación con el número de egresados, y carencia de un organismo que ofrezca información confiable y que asegure la calidad de los estudios de postgrado.

Para contribuir a mejorar esta situación, el CONCYTEC, en su calidad de órgano promotor y coordinador del desarrollo científico y tecnológico, cuenta con diversos programas de apoyo (becas para estudios de postgrado, ayudas a la investigación, proyectos especiales, etc.), y gestiona y orienta fondos de ayuda a la investigación y a la formación de postgrado de acuerdo con las necesidades del país y con al progreso científico-tecnológico.

Con el fin de desempeñar de manera apropiada esta misión, el CONCYTEC necesita, entre otras cosas, desarrollar una estrategia para el mejoramiento de la calidad de la formación y de la investigación en función de las necesidades del país y de los estándares internacionales. Siendo la enseñanza de postgrado la vía usual para la formación de investigadores y el entorno en el que se desarrolla buena parte de la investigación, es lógico que el CONCYTEC promueva el mejoramiento de la calidad de las instituciones y de los programas que imparten formación de postgrado.

Después de hacer un diagnóstico de la situación y de la calidad de los estudios de postgrado en áreas consideradas como prioritarias para el desarrollo nacional en el actual contexto de globalización, el CONCYTEC ha creado recientemente el Programa de Promoción y Evaluación de la Calidad de los Estudios de Postgrado ( PECEP). Este Programa se propone proveer de criterios, de instrumentos operativos y de información precisa al CONCYTEC, y, en general, a los organismos que apoyan la investigación y la formación de al to nivel, para la toma de decisiones con respecto a sus políticas, a las estrategias y a las acciones de apoyo a grupos de investigación y de formación de postgrado. A partir de este objetivo general, el PECEP Ilevará a cabo una política participativa y una estrategia de mejoramiento de la calidad de los estudios de postgrado, orientadas a promover la cultura de la calidad y a llevarla a la práctica a través de mecanismos concretos de autoevaluación.

Para promover la cultura de la calidad de los estudios de postgrado en áreas consideradas prioritarias, el programa PECEP ha 
desarrollado cuatro talleres regionales de trabajo: Arequipa, Huancayo, Pucallpa y Trujillo, con escuelas de postgrado y con institutos de investigación, a fin de aglutinar información sobre la situación de la investigación y de los estudios de postgrado, de facilitar las conexiones y los intercambios, de optimizar los contenidos, los procesos y la institucionalidad de la gestión, y de elaborar lineamientos para mejorar la calidad de los estudios de postgrado. Como culminación de esta primera etapa, y con el apoyo de la OEI, se realizó en Lima (25-28 de agosto de 2004) el seminario «La investigación y los postgrados en ciencia y tecnología en el Perú». Un informe pormenorizado que publicará próximamente la Revista Iberoamericana de Educación (RIE), dará cuenta del desarrollo del PECEP y de los avances que ha realizado.

Pero no sólo le preocupa al CONCYTEC mejorar la calidad de los estudios de postgrado. La ANR y algunas universidades también están empeñadas en iniciar procesos de evaluación y acreditación en las escuelas de postgrado.

La ANR formó en el 2002 una comisión técnica para elaborar una Propuesta de Acreditación de Programas de Postgrado. Tal propuesta fue enviada en mayo de ese año a todas las universidades, pidiéndoles su opinión. Sólo una privada de reciente creación contestó, y, en junio de 2003, la ANR remitió otra vez el documento a todas las universidades.

También en dicho mes, y para su consulta, la ANR dirigió a las universidades el documento Requisitos mínimos para la organización y funcionamiento de una escuela de postgrado o sección de postgrado. En ese documento se indica un conjunto de requisitos necesarios, tanto cualitativos como cuantitativos para el funcionamiento de una escuela de postgrado, tales como organización, planes de estudio, facilidades para el uso de bibliotecas y de laboratorios, y número y calidad de docentes.

Por su parte, en 1997 la Sección de Postgrado de la Facultad de Ciencias de la Universidad Nacional de Ingeniería pidió a la Asociación Universitaria Iberoamericana de Postgrado ( AUIP), de la cual la UNI es miembro (como también lo son las universidades Católica del Perú, Católica Santa María de Arequipa, de Lima, de Piura, Ricardo Palma y San Marcos), una evaluación de su programa de doctorado cooperativo en Física, que cuenta con el apoyo de la cooperación internacional (sobre todo de la U niversidad sueca de U ppsala), y que tuvo su primer graduado (doctor en Física) en 1990. Esta evaluación fue realizada por dos 
expertos de la AUIP, uno de España y otro de Colombia, que incluyó una visita a la UNI de una semana en noviembre de 1997, y que concluyó con un amplio informe, todavía inédito, que indicaba que los estudios de doctorado son de buena calidad, que los graduados tienen un alto nivel, mas que el programa es básicamente resultado del esfuerzo de pocos docentes, aun tomando en consideración el apoyo de la cooperación internacional, pero que no muestra el respaldo ni la institucionalización requeridos para ameritar una acreditación. Con esta evaluación, cuyo resultado era previsible para los docentes involucrados, se buscaba obtener un mayor apoyo al programa por parte de la UNI, especialmente económico, lo que se ha logrado, pero de forma muy limitada.

La Escuela de Postgrado de la Universidad Peruana Cayetano Heredia elaboró, en septiembre de 2002 , el documentoHacia un modelo de autoevaluación de calidad de programas de postgrado, inspirado a su vez en el documentoEvaluación y acreditación de programas de postgradoGuía de autoevaluación, de la AUIP, y que es hoy la base para una autoevaluación de las escuelas de postgrado del ya mencionado Consorcio de Universidades.

\section{LEGISLACIÓN SOBRE ACREDITACIÓN}

La legislación sobre evaluación y acreditación de estudios superiores, si exceptuamos lo relativo a las facultades y escuelas de Medicina (parágrafo 2), es aún muy reciente.

En el Perú la educación superior, sea pública o privada, comprende la superior no universitaria y la universitaria. La primera se rige por la Ley General de Educación (LGE), y es impartida por los institutos superiores tecnológicos, por los institutos superiores pedagógicos, por las escuelas nacionales de artes, y por otros centros de formación superior. La segunda se rige por una ley específica, la $n .023733$, que fue promulgada en diciembre de 1983, y que es conocida como ley universitaria. Después de esa fecha se aprobaron algunas normas que modificaron aspectos concretos de la ley universitaria. Es importante anotar que, por disposición de la Constitución, cada universidad es autónoma. La Asamblea Nacional de Rectores (ANR), que las comprende a todas, es una instancia de coordinación interuniversitaria. Existe, además, el Consejo Nacional para la Autorización del Funcionamiento de Universidades (CONAFU), cuyas atribuciones sólo se refieren a la licencia inicial 
de funcionamiento de universidades nuevas y a su seguimiento durante los primeros cinco años.

La ley universitaria vigente, la ya citada $n .023733$, no dice nada sobre acreditación ni sobre evaluación; lo único que establece (artículo 25) es que las universidades están obligadas a mantener sistemas de evaluación internos para garantizar la calidad de sus graduados y de sus profesionales. La ausencia de normativas precisas ha hecho que la calidad de la oferta de educación superior quede librada a la voluntad y al buen criterio de las propias universidades.

Esta situación ha sido corregida por la nueva LGE, la n. 028044 , que fue promulgada en julio de 2003, pero cuyo reglamento aún (agosto 2004) no ha sido aprobado.

Con respecto al mejoramiento de la calidad educativa y a la introducción de la acreditación, la LGE introduce como principios de la educación peruana «la calidad, que asegura condiciones adecuadas para una educación integral, pertinente, abierta, flexible y permanente» (art. 8. ${ }^{\circ}$ inc. «d»); crea el Sistema Nacional de Evaluación, Acreditación yCertificación de la Calidad Educativa (art. $14 .^{\circ}$ ) con los organismos que lo componen: el Instituto Peruano de Evaluación, Acreditación y Certificación Educativa, para educación básica, y con «un organismo que será creado y normado por ley específica», para educación superior (art. $\left.15 .^{\circ}\right)$; y establece como funciones de estos organismos las siguientes (art. $\left.16 .{ }^{\circ}\right)$ :

a) Promover una cultura de la calidad entre los docentes y las instituciones educativas.

b) Evaluar, en los ámbitos nacional y regional, la calidad del aprendizaje y de los procesos pedagógicos y de gestión.

c) Acreditar, periódicamente, la calidad de las instituciones educativas públicas y privadas.

d) Certificar y recertificar las competencias profesionales.

e) Difundir los resultados de las acciones evaluadoras y acreditadoras de las instituciones educativas, haciendo uso de los medios de comunicación.

f) Desarrollar programas orientados a formar profesionales especializados en evaluar logros y procesos educativos. 
g) Compatibilizar los certificados, los grados, los diplomas y los títulos educativos nacionales, y establecer su correspondencia con certificaciones similares expedidas en el extranjero.

h) Elaborar, con participación de las instancias descentralizadas, los indicadores de medición de la calidad que contribuyan a orientar la toma de decisiones.

Por otro lado, la mencionada LGE (art. $21 .^{\circ}$ ) establece que el Estado promueve la universalización, la calidad y la equidad de la educación, asignándole, entre otras, las siguientes funciones:

a) Ejercer un rol normativo, promotor, compensador, concertador, articulador, garantizador, planificador, regulador y financiador de la educación nacional.

c) Promover el desarrollo científico y tecnológico en las instituciones educativas de todo el país, y la incorporación de nuevas tecnologías en el proceso educativo.

d) Reconocer e incentivar la innovación y la investigación que realizan las instituciones públicas y privadas.

h) Ejercer y promover un proceso permanente de supervisión y de evaluación de la calidad y de la equidad en la educación.

i) Informar y rendir cuentas, ante los usuarios y ante la población, de la situación y del cumplimiento de los objetivos y de las metas de la educación.

j) Supervisar y evaluar las acciones de educación, de cultura y de recreación a nivel nacional, regional y local.

Desde la promulgación de la LGE, tanto el Ministerio de Educación como la ANR, algunos colectivos de educadores y los propios congresistas, han elaborado propuestas de reglamentación referidas unas a la totalidad de la ley, y otras a los artículos específicos relativos a la evaluación y a la acreditación.

Con respecto al Sistema Nacional de Evaluación, Acreditación y Certificación de la Calidad Educativa (SINEACE) al que se refiere el artículo $14 .^{\circ}$ de la Ley General de Educación, se propone que sea un sistema único, porque articula los procesos para el mejoramiento de la calidad en todo el territorio nacional; flexible, porque diseña su política 
respetando las particularidades de las instituciones educativas o de las personas que se encuentran dentro del ámbito de su competencia; e integral, porque abarca los diferentes factores que inciden en la calidad de la educación. Sus organismos operadores serán el Instituto Peruano de Evaluación, Acreditación y Certificación Educativa (IPEACE) para la educación básica y la técnico-productiva, y un organismo creado y normado por ley específica para la superior.

La propuesta del Ministerio de Educación con respecto al IPEACE entiende este instituto como un organismo de derecho público, con autonomía técnica, administrativa y funcional para el ejercicio de sus funciones respecto del Sector Educación, que cuenta con una partida presupuestaria específica. Además, sugiere que el IPEACE establezca relaciones de articulación con el organismo de evaluación, acreditación y certificación de la calidad de la educación superior, con miras a que el diseño de las políticas generales que dirijan las acciones educativas en el ámbito de la educación superior guarden coherencia y tengan como base fundamental la calidad educativa de la educación básica y de la educación técnico-productiva.

En relación con la reglamentación de lo relativo a la acreditación de la educación superior, también hay varios proyectos en la Comisión de Educación del Congreso de la República. Uno de ellos, que cuenta con un cierto consenso en la comunidad educativa, entiende el Consejo Nacional de Evaluación, Acreditación y Certificación de la Calidad de la Educación Superior (CONEACES) como un organismo autónomo dotado de un régimen legal y administrativo que garantiza su independencia. Es una persona jurídica de derecho público que opera de manera desconcentrada e intersectorial, y que está adscrito al Sector Educación. Entre sus objetivos señala: contribuir al diseño de las políticas de evaluación, acreditación y certificación de la calidad de la enseñanza superior; garantizar la calidad de las instituciones de educación superior y fomentar en ellas procesos permanentes de mejoramiento de la calidad académica y de gestión, articulando la educación con las necesidades de la sociedad y la responsabilidad de ésta en su función educadora; propiciar que los procesos de creación de las instituciones de educación superior respondan a las necesidades de la sociedad; garantizar que la prestación de servicios profesionales se ofrezca al nivel o por encima de los estándares mínimos de calidad previamente establecidos; promover la integración de la educación superior identificando las diferencias y las complementariedades de las instituciones de educación superior universitarias y no universitarias; y contribuir al diseño de las 
políticas de desarrollo nacional proporcionando la información obtenida durante el ejercicio de sus funciones.

Como puede comprobarse por lo dicho hasta ahora, el Perú ya cuenta con una Ley General de Educación que introduce la evaluación y la acreditación de la educación, y, en especial, las de la educación superior. Sin embargo, todavía no se han aprobado los reglamentos relativos a los artículos de esta ley que tienen que ver con la evaluación y la acreditación. Se espera que en los próximos meses dichos reglamentos sean aprobados, y que comiencen oficialmente los procesos de evaluación, acreditación y certificación.

A pesar de la falta de reglamentos, lo cierto es que la ley ha dado un significativo impulso a la difusión de la cultura de la calidad y la acreditación. De hecho, las universidades están embarcadas en el montaje de oficinas de evaluación, y en el diseño y elaboración de los materiales y de las herramientas necesarios para iniciar los procesos de autoevaluación, de evaluación externa y de acreditación.

\section{BIBLIOGRAFÍA}

ABELEDO, C. L. (2003): Investigación en universidades y formación de recursos humanos en disciplinas seleccionadas, Informe de consultoría, Lima, ProyectoconCYTECBID.

Asamblea NaCional de Rectores (ANR) $(2002,2003,2004)$ : Actas de sesiones de la Comisión Nacional de Rectores para la Acreditación, Lima, ANR.

- (2002): Propuesta de acreditación de programas de postgrado (maestría y doctorado), Lima, ANR.

- (2003): Requisitos mínimos para la organización y funcionamiento de una escuela de postgrado o sección de postgrado, Lima, ANR.

ASOCIACIÓN U NIVERSITARIAl BEROAMERICANA DE POSTGRADO(AUIP) (1997): Evaluación externa y acreditación internacional de programas de postgrado y doctorado: pautas y lineamientos generales, Salamanca, AUIP.

- (1997) Evaluación y acreditación de programas de postgrado. Guía de autoevaluación, Salamanca, AUIP.

- (1997): Evaluación del doctorado de Física de launı. Documento interno, Lima, UNI. 
COMISIÓN DE ACREDITACIÓN DE FACULTADES O EsCUELAS de Medicina HumANa (CAFME) (2001): Ley, normas de ejecución yestándares mínimos para la acreditación de facultades o escuelas de Medicina, Lima, CAFME.

- (2002): Boletín CAFME, año 1, n.ำ1, Lima, CAFME.

- (2002): Acreditación de facultades o escuelas de Medicina. Ley, reglamento, estándares mínimos de acreditación y manual de procedimientos, Lima, CAFME.

- (2002): Boletín CAFME, año 1, n. 3, Lima, CAFME.

- (2003): Informe sobreel proceso de Acreditación de Facultades o Escuelas de Medicina. Comunicado n. 0002 . Lima, CAFME, 2003.

- (2003): Boletín CAFME. año 2, n.o 4, Lima, CAFME.

Congreso de la Repúblicadel Perú.C omisión deE ducación, Cienciay TeCnología (2003): Proyecto de Ley de Educación Superior Universitaria.

- (2004): Proyecto de Ley para regular el Consejo Nacional de Evaluación, Acreditación y Certificación de la Calidad de la Educación Superior.

- (2004): Proyecto de Ley para regular el Sistema Nacional de Evaluación, Acreditación y Certificación de la Calidad de la Educación.

- (2004): Proyecto de Ley sobre el Instituto Peruano de Evaluación, Acreditación y Certificación de la Calidad Educativa, IPEACE.

Consej o nacional de Cienciay Tecnología (2003): Propuesta de programa de promoción y acreditación de la calidad de los estudios de postgrado, Lima, CONCYTEC.

- (2004): Programa de Promoción yE valuación de la Calidad de los Estudios de Postgrado, PECEP, Lima, CONCYTEC, 2004.

ESTADO PERUANO (1983): Ley (universitaria) n. 23733, diciembre de 1983.

- (2002): Ley orgánica del Consejo Nacional de Ciencia y Tecnología. Decreto Legislativo $n .0112,6 / 6 / 1981$ y modificaciones: Ley $n .{ }^{\circ} 27690,8 / 3 / 2002$.

- (2003): Ley General de Educación n.ำ28044, 28/7/2003.

- (2004): Ley marco de ciencia, tecnología e innovación tecnológica $n . \stackrel{0}{0}$ $28303,27 / 7 / 2004$.

Grobman, A. (2003): Estado de la investigación en las ciencias agrarias en el Perú. Informe de consultoría, Lima, Proyecto CONCYTEC-BID.

Guerra-García, R. (2002): Estudios de postgrado e investigación. Conferencia magistral pronunciada en el foro «Hacia una nueva Ley Universitaria», Lima, UNMSM.

Gutiérrez, M. (2003): Biología, Bioquímica y Biología Molecular. Capacidad instalada. Universidades y sus programas de investigación. Informe de consultoría, Lima, Proyecto CONCYTEC-BID. 
Heraud, J. A. (2003): Relevamiento e identificación de demandas para los componentes del Programa de Ciencia y Tecnología: proyectos de investigación. Informe de consultoría, Lima, Proyecto CONCYTEC-BID.

NAVA, H. L. (2003): «Evaluación y Acreditación de la Educación Superior: el caso del Perú», en: Seminario-Taller Internacional: Evaluación y Acreditación de la Educación Superior en América Latina y el Caribe, Buenos Aires, CONEAU-IESALC, 2003. Boletín de IESALC-UNESCO, Buenos Aires, n. 047 .

Piscoya ARBAÑIL, J . L. (2002): «CAFME: Historia, perspectiva actual y futura», en Boletín CAFME, 1, 1, p. 10, Lima, Ministerio de Salud, Comisión de Acreditación de Facultades o Escuelas de Medicina Humana (CAFME).

SOTA N ADAL, J. (2003): «El sentido de la segunda reforma universitaria en el Perú», Boletín de IESALC-UNESCO, Buenos Aires, n. ${ }^{\circ} 50$.

- (2003): Relevamiento e identificación de demandas para el componente 2 del Programa de Ciencia y Tecnología: fortalecimiento y creación de capacidades. Informe de consultoría, Lima, Proyecto CONCYTEC-BID.

UNIVERSIDADN ACIONAL DE I NGENIERÍA. OFICINA DE ACREDITACIÓN YCALIDAD UNIVERSITARIA (2003): Colección de publicaciones sobre acreditación universitaria. 7 vols.

- (2003): Formato para preevaluación, usando los estándares mínimos para la acreditación, Lima, UNI.

Universidad Peruana Cayetano Heredia (UPCH) (2001): «Situación actual y perspectivas de los postgrados», en Diálogos universitarios de postgrado, vol. 2, Escuela de Postgrado Víctor Alzamora Castro, Lima, UPCH.

- (2002): «Autoevaluación y acreditación de postgrados: conceptos y experiencias», en Diálogos universitarios de postgrado, vol. 6, Escuela de Postgrado Víctor Alzadora Castro, Lima, UPCH.

- (2002): Hacia un modelo de autoevaluación de calidad de programas de postgrado, Escuela de Postgrado Víctor Alzadora Castro, Lima, UPCH. 


\title{
Contactar
}

Revista lberoamericana de Educación

\author{
Principal OEI
}

